according to UCEIS score and additional TW criteria on day 0 (figure 1).

UCEIS score $>6$ predicted higher need for rescue therapy (Chi square, $\mathrm{p}=0.01$ ) but not colectomy during same admission $(p=0.68)$ or within 1 year $(p=0.41)$.In logistic regression analysis, UCEIS predicted rescue therapy $(\mathrm{p}=0.01)$ but not colectomy during same admission $(p=0.68)$ or within 1 year $(p=0.55)$; whereas day 0 TW criteria predicted need for rescue therapy $(p=0.02)$, colectomy during admission $(p=0.04)$ and within 1 year $(p=0.03)$. D3 response predicted colectomy during same admission $(\mathrm{p}=0.001)$ and within 1 year $(\mathrm{p}=0.0002)$.

Conclusion Endoscopic severity predicts use of rescue therapy but not colectomy rates (During same admission and at 1 year) whereas biological severity predicts use of rescue therapy, colectomy during same admission and at 1 year. Clinical criteria assessed by D3 response are the strongest predictors of colectomy on that admission or within 1 year.

\section{P107 CHANGING OUTCOMES IN ACUTE SEVERE ULCERATIVE COLITIS AT OXFORD IN LAST SEVEN DECADES}

Vipin Gupta*, Waled Mohsen, Alex Adams, Aisling Murphy, Dharshan Sathananthan, Sarah Cripps, Oliver Brain, Rebecca Palmer, Tim Ambrose, Bruce George, Alissa Walsh, Simon Travis, Jack Satsangi. Translational Gastroenterology Unit, John Radcliffe Hospital, Ouh, Oxford, UK

\subsection{6/gutjnl-2020-bsgcampus. 182}

Introduction Acute severe ulcerative colitis (ASUC) is a common medical emergency, with up to $25 \%$ of patients with ulcerative colitis experiencing at least one severe attack in their life-time. Since first Randomized control of efficacy of intravenous steroids published in 1954, many drugs have been discovered and used in management of acute severe colitis either as rescue therapy(Ciclosporin/Infliximab) or maintenance agents( 5-ASA, Immunomodulator (Azathioprine/6-Mercaptopurine), Biologic( Infliximab/Adalimumab/Vedolizumab) and small molecules( Tofacitinib). What is not known if these drugs have materialized into better outcomes in acute severe colitis. Methods We analysed outcomes from different papers from Oxford representing different cohorts of different era i.e Dinesen et al(1953-2007), Travis et al( 1996) and Corte et al( 2015). We compared the outcomes with most recent cohort from 2015-2019.
Results Consecutive 131 admissions (117 patients) between 2015-9 were analysed. All satisfied modified TW definition of ASUC. Sixty-eight patients (58\%) were female, index presentation 38 (29\%), median age at presentation 40 years (16-76), median disease duration 1 year (1-43), median follow up 23 months (1-49).Seventy-one (54\%) received rescue therapy (ciclosporin 35/71 and anti-TNF 36/71).Colectomy rates were $15 \%(19 / 131)$ during same admission and 26\% (30/117) within 1 year of follow up.

We compared the outcomes in different cohorts. We observed that colectomy rates have been decreasing significantly with better treatment (Image 1). We also observed that readmissions with acute severe colitis have also reduced (better maintenance) with only $12 \%$ patients requiring readmission in first year. Seventy percent of patients in current cohort have been maintained on biologic or Tofacitinib leading to colectomy free survival for median follow up of 2 years.

Conclusion Availability of multiple drug options and improvement in healthcare have led to improved outcomes in acute severe colitis justifying the cost associated with these drugs.

\section{P108 NATIONAL MICROSCOPIC COLITIS DISEASE REGISTRY: VARIATIONS IN PATIENT JOURNEY}

${ }^{1}$ Nicole Haggarty*, ${ }^{2}$ Christopher Kelly, ${ }^{3}$ Ruridh Allen, ${ }^{4}$ Rebecca Grant, ${ }^{4}$ Andrew Robertson, ${ }^{4}$ William Brindle, ${ }^{4}$ Alexander Robertson, ${ }^{2}$ Josh Orpen-Palmer, ${ }^{2}$ Jennifer Veryan, ${ }^{4}$ Paul Fineron, ${ }^{2}$ Aidan Cahill, ${ }^{3}$ Hasnain Jafferbhoy, ${ }^{2}$ John Paul Seenan, ${ }^{4}$ Tassos Koulaouzidis, ${ }^{4}$ Ian Arnott, ${ }^{1}$ Santosh Salunke. ${ }^{1}$ NHS Forth Valley, UK; ${ }^{2}$ NHS Greater Glasgow and Clyde, UK; ${ }^{3}$ NHS Fife, UKi ${ }^{4}$ NHS Lothian, UK; ${ }^{5}$ Scottish Society of Gastroenterology, UK

\subsection{6/gutjnl-2020-bsgcampus.183}

Introduction Microscopic colitis (MC) is still perceived to be an 'uncommon' condition ${ }^{1}$. Despite significant impact on quality of life $^{1}$, many aspects of the patient journey remain unclear.

A National MC Disease Registry is being developed with the aim of gathering data on epidemiology, variations in clinical practice and patient journey. The secondary aim is to generate academic and clinical data to help create more streamlined MC Services, improving patient care and outcomes.

Methods Retrospective data was collected across 6 Scottish (2 DGHs/4 University teaching) units. Once identified from Pathology databases, further data was collected from electronic records. 